\title{
Video Article \\ An Air-liquid Interface Bronchial Epithelial Model for Realistic, Repeated Inhalation Exposure to Airborne Particles for Toxicity Testing
}

\author{
Hedwig M. Braakhuis ${ }^{1}$, Ruiwen He ${ }^{1,2}$, Rob J. Vandebriel ${ }^{1}$, Eric R. Gremmer ${ }^{1}$, Edwin Zwart ${ }^{1}$, Jolanda P. Vermeulen ${ }^{1}$, Paul Fokkens ${ }^{1}$, John Boere ${ }^{1}$, \\ Ilse Gosens ${ }^{1}$, Flemming R. Cassee $e^{1,2}$ \\ ${ }^{1}$ National Institute for Public Health and the Environment (RIVM) \\ ${ }^{2}$ Institute for Risk Assessment Sciences (IRAS)
}

Correspondence to: Hedwig M. Braakhuis at hedwig.braakhuis@rivm.nl

URL: https://www.jove.com/video/61210

DOI: doi: $10.3791 / 61210$

Keywords: Biology, Issue 159, air-liquid interface, bronchial model, inhalation exposure, toxicity, realistic exposure, in vitro, nanomaterials

Date Published: 5/13/2020

Citation: Braakhuis, H.M., He, R., Vandebriel, R.J., Gremmer, E.R., Zwart, E., Vermeulen, J.P., Fokkens, P., Boere, J., Gosens, I., Cassee, F.R. An Air-liquid Interface Bronchial Epithelial Model for Realistic, Repeated Inhalation Exposure to Airborne Particles for Toxicity Testing. J. Vis. Exp. (159), e61210, doi:10.3791/61210 (2020).

\section{Abstract}

For toxicity testing of airborne particles, air-liquid interface (ALI) exposure systems have been developed for in vitro tests in order to mimic realistic exposure conditions. This puts specific demands on the cell culture models. Many cell types are negatively affected by exposure to air (e.g., drying out) and only remain viable for a few days. This limits the exposure conditions that can be used in these models: usually relatively high concentrations are applied as a cloud (i.e., droplets containing particles, which settle down rapidly) within a short period of time. Such experimental conditions do not reflect realistic long-term exposure to low concentrations of particles. To overcome these limitations the use of a human bronchial epithelial cell line, Calu-3 was investigated. These cells can be cultured at ALI conditions for several weeks while retaining a healthy morphology and a stable monolayer with tight junctions. In addition, this bronchial model is suitable for testing the effects of repeated exposures to low, realistic concentrations of airborne particles using an ALI exposure system. This system uses a continuous airflow in contrast to other ALI exposure systems that use a single nebulization producing a cloud. Therefore, the continuous flow system is suitable for repeated and prolonged exposure to airborne particles while continuously monitoring the particle characteristics, exposure concentration, and delivered dose. Taken together, this bronchial model, in combination with the continuous flow exposure system, is able to mimic realistic, repeated inhalation exposure conditions that can be used for toxicity testing.

\section{Introduction}

The lungs are vulnerable to inhalation exposure to airborne particles. To assess the potential toxicity of airborne particles, progress has been made to develop air-liquid interface (ALI) exposure systems ${ }^{1,2,3,4,5}$. ALI exposure systems allow more relevant and realistic exposure models compared to traditional submerged exposure via culture medium that alters the characteristics and kinetics of the particles ${ }^{6}$. The ALI exposure systems place specific demands on the cell culture models, as the models lack culture medium and thus nutrients at the apical side. Many cell models are negatively affected by being cultured and exposed at the air (e.g., drying out) and only remain viable for a few days. This limits the exposure conditions that can be used in these models: usually relatively high concentrations are applied within a short period of time as a cloud (i.e., droplets containing particles, which settle down rapidly). Such experimental conditions do not reflect realistic long-term exposure to low concentrations of particles; thus, the relevance of the results can be questioned. To overcome these limitations, the culture and exposure protocol for a bronchial model consisting of the human bronchial epithelial cell line Calu- $3^{7}$ was optimized.

Most in vitro lung models used for ALI exposure contain other cell lines such as A549, BEAS-2B, and 16HBE140- (16HBE) or primary cells as a basis $^{8}$. These cell lines have the disadvantage that they remain viable for only a few days when cultured at the ALI. In addition, some of these cell lines overgrow when cultured for a period longer than 5 days. Finally, A549 cells miss functional tight junctions and can therefore not form a tight barrier that is needed to mimic the lungs ${ }^{9,10}$. Primary epithelial cells might be a good option for ALI exposure as they can be cultured at the ALI for weeks. However, primary cells differ from batch to batch, are more difficult to maintain, and are more expensive compared to cell lines, which makes them less suitable for toxicity testing and screening. When comparing different human bronchial epithelial cell lines (16HBE, Calu-3, H292, and BEAS-2B), only the Calu-3 cells fulfill all criteria needed for realistic, repeated ALI exposure: they remain viable for weeks while cultured at the ALI, provide a high barrier integrity, do not overgrow, and are easy to culture and maintain. Calu- 3 cells originate from an adenocarcinoma and are able to produce mucus ${ }^{11,12}$. There are inconsistencies as to whether the cells can develop cilia ${ }^{11,13}$. Calu-3 cells are also a suitable model to study respiratory syncytial virus (RSV) infections that infect ciliated airway epithelial cells ${ }^{14}$.

Besides the cell model, an automated exposure system (AES) is used for the air-liquid exposure to aerosols ${ }^{15,16}$. The AES has the advantage that it uses a continuous airflow to expose the cell model to aerosols. This is in contrast to other air-liquid exposure systems that usually use relatively high concentrations within a short period of time as a cloud (i.e., droplets containing particles that settle down rapidly) ${ }^{17,18,19}$. These cloud systems do not reflect realistic long-term exposure to low concentrations of particles. By applying a continuous airflow using the AES, the cell model can be exposed to a low concentration of particles over a longer time period, reflecting realistic exposure conditions. Another advantage over cloud systems is that the AES has the option to connect particle characterization instruments, allowing measurement of particle 
size, number concentration, and mass over time. A limitation of the AES is that it uses relatively high airflows between $10 \mathrm{~mL} / \mathrm{min}$ and $100 \mathrm{~mL} /$ $\min$.

\section{Preparing cell culture medium (CCM)}

1. Prepare a bottle of $500 \mathrm{~mL}$ of minimum essential medium (MEM) supplemented with glutamine.

2. Add $5 \mathrm{~mL}$ of penicillin-streptomycin (i.e., $100 \mathrm{U} / \mathrm{mL}$ penicillin and $100 \mu \mathrm{g} / \mathrm{mL}$ streptomycin).

3. Add $5 \mathrm{~mL}$ of non-essential amino acids (NEAA) solution.

4. Add $10 \mathrm{~mL}$ of amphotericin $\mathrm{B}$ (optional).

5. Add $50 \mathrm{~mL}$ of FBS (heat inactivated, please follow the ATCC protocol for heat inactivation; (https://www.atcc.org/ /media/PDFs/Culture \%20Guides/AnimCellCulture_Guide.ashx, page 19)).

\section{Subculturing of Calu-3 cells}

NOTE: Calu- 3 cells are cultured in T75 or T175 cell culture flasks at $37{ }^{\circ} \mathrm{C}$ and $5 \% \mathrm{CO}_{2}$. Cells are passaged at $60-80 \%$ confluency every 7 days with CCM renewal every 2-3 days. CCM is poured off and fresh CCM (T25 = $5 \mathrm{~mL}, \mathrm{~T} 75=15 \mathrm{~mL}$, and T175 $=25 \mathrm{~mL}$ ) is pipetted into the flask and the flask is placed back into the incubator. Cells should be passaged at least $2 x$ after thawing, before using in experiments, or before freezing, and they should be passaged no more than $25 \mathrm{x}$ in total.

1. Confirm if flask is $60-80 \%$ confluent by checking under a light microscope.

2. Pour off the CCM from the flask.

3. Wash the cells $2 x$ with $5 \mathrm{~mL}$ of $1 x$ Hanks' Balanced Salt Solution (HBSS) without calcium and without magnesium. Discard the HBSS after each wash. HBSS removes serum, which inhibits trypsin.

4. Add $3 \mathrm{~mL}$ of trypsin-EDTA for a T75 (4 mL for a T175) and place the flask back into the incubator at $37{ }^{\circ} \mathrm{C}$ and $5 \% \mathrm{CO}_{2}$ for $10-15 \mathrm{~min}$. Check after $10 \mathrm{~min}$, ensuring the cells have become detached from the flask surface. In case the cells are grown to $>80 \%$ confluency, they will not detach using trypsin $0.05 \%$ and trypsin $0.25 \%$ could be used.

5. Add $6 \mathrm{~mL}$ (i.e., double the trypsin-EDTA volume originally added) of CCM to the flask and gently rock the flasks to ensure proper mixing. This is to ensure the trypsin has been neutralized by the FBS in the CCM and its activity on the cells halted. If trypsin is allowed to remain in contact with the cells for too long they will not reattach when put into a new cell culture flask.

6. Pour the complete contents of the flask into a $50 \mathrm{~mL}$ centrifuge tube.

7. Centrifuge the cells for 5 min at $130 \times \mathrm{g}$, ensuring that the centrifuge is correctly balanced.

8. Return the vial containing the cells back to aseptic conditions and remove the supernatant gently, without disturbing the pellet. The supernatant can be poured off and the remainder pipetted off, ensuring the pellet is not disturbed.

9. Resuspend the cell pellet in $1 \mathrm{~mL}$ of CCM by pipetting up and down until all cells are suspended (i.e., no pellet or cell agglomerates are observed). Additional CCM can be added to dilute the cell suspension.

10. Count the cells, both dead and alive, in $1 \mathrm{~mL}$ of CCM using a hemocytometer. If needed for proper counting, dilute the cells in 3 or $4 \mathrm{~mL}$.

11. Suspend the cells into the CCM volume required and add the cell suspension into each flask. To achieve about $80 \%$ confluency in a week, seed $2 \times 10^{6}$ cells in a T75 or $6 \times 10^{6}$ cells in a T175.

12. Gently rock the flask and then place it back into the incubator at $37{ }^{\circ} \mathrm{C}$ and $5 \% \mathrm{CO}_{2}$.

13. Replace with fresh CCM every $2-3$ days and subculture when the cells reach $60-80 \%$ confluency.

\section{Seeding Calu-3 cells onto culture inserts}

NOTE: Inserts are available with different pore sizes. Small pore sizes (e.g., $0.4 \mu \mathrm{m}$ ) have the advantage that the cells grow more easily and can achieve a good barrier already after 5 days culturing under submerged conditions, as measured by Trans Epithelial Electrical Resistance (TEER). However, when interested in particle translocation, these pores are too small and will trap the particles. Therefore, larger pore sizes (e.g., $3 \mu \mathrm{m}$ ) are usually chosen to test particles. When using a larger pore size, the cells need longer time periods (e.g., 7-10 days culturing under submerged conditions) to achieve a good TEER.

1. Prepare cell suspension with known concentration following steps 2.1-2.10.

2. Dilute cells to a concentration of $5 \times 10^{5}$ cells $/ \mathrm{mL}$ in prewarmed CCM for 6 well inserts or $2.5 \times 10^{5}$ cells $/ \mathrm{mL}$ for 12 well inserts.

3. Take a cell culture plate with inserts and place under aseptic conditions.

4. Fill the basolateral side with $2 \mathrm{~mL}$ of prewarmed CCM for 6 well inserts, or $1 \mathrm{~mL}$ for 12 well inserts. While adding the culture medium, take the insert out using tweezers.

5. Carefully mix the cell suspension by pipetting up and down. Pipette $1.0 \mathrm{~mL}$ for 6 well inserts and $500 \mu \mathrm{L}$ for 12 wells inserts (equivalent to 100,000 cells $/ \mathrm{cm}^{2}$ ) on the top of the membrane in the cell culture insert with $0.4 \mu \mathrm{m}$ pores. For $3.0 \mu \mathrm{m}$ pores, increase the cell density to $128,000 \mathrm{cell} / \mathrm{cm}^{2}$

6. Cover the cell culture plate and incubate at $37^{\circ} \mathrm{C}$ and $5 \% \mathrm{CO}_{2}$.

7. Change the CCM every $2-3$ days.

8. Let the cells become confluent for 7 days under submerged conditions before continuing to culture at the ALI.

9. Measure TEER.

1. Take an Epithelial Voltohmmeter supplemented with Chopstick Electrode Set and charge the battery system overnight.

2. Disconnect the Voltohmmeter from the charger and connect the chopstick electrode.

3. Clean the electrode with $70 \%$ ethanol. 
4. Place the electrode in the CCM by putting the longer electrode in the external culture media until it touches the bottom of the dish and putting the shorter electrode in the media without touching the membrane.

5. Start with an empty insert without cells. Wait until the measurement stabilizes and write down the resistance in Ohms. This measurement is the resistance of the insert membrane without any cells (i.e., blank resistance)

6. Repeat the measurement for each insert and subtract the blank resistance to obtain the true resistance.

7. For data analysis, multiply the resistance values by the surface area of the insert into $\Omega \times \mathrm{cm}^{2}$. For a 6 well insert, the surface area is $4.67 \mathrm{~cm}^{2}$. Thus, if a resistance of $600 \mathrm{Ohm}$ is measured and the background is $120 \mathrm{Ohm}$, the resistance is $480 \mathrm{Ohm}$, which is then multiplied by the surface area of $4.67 \mathrm{~cm}^{2}$ for a total of $2,241.6 \mathrm{Ohm} \mathrm{x} \mathrm{cm}{ }^{2}$. The TEER should be $>1,000 \Omega \times \mathrm{cm}^{2}$ to continue.

10. Remove the CCM from the apical side of the inserts.

11. Add $2 \mathrm{~mL}$ of prewarmed CCM for 6 well and $1 \mathrm{~mL}$ for 12 well inserts to the basolateral side of the well (i.e., under the cell culture insert). The CCM should touch the membrane from the bottom, but not leak onto the top of the insert

12. At this point cells are apically exposed to air, which is referred to as culturing at the ALI.

13. Culture cells at the $\mathrm{ALI}$ in the incubator at $37^{\circ} \mathrm{C}$ and $5 \% \mathrm{CO}_{2}$ for 7 days prior to exposure.

14. Change the basolateral CCM every 2-3 days. The cells can be used at the ALI for 6 weeks.

\section{Preparing the exposure setup}

NOTE: Sections 5-7 describe preparations for particle exposure using an automated exposure station (AES, see Table of Materials). The setup for particle nebulization and characterization is also compatible with other ALI exposure systems from other manufacturers. As an example, the exposure to particles is described below. Such systems can also be used for other exposures, such as sensitizers, cigarette smoke, and diesel exhaust. Figure 1 shows the AES and an exposure module. Figure 2 shows a schematic representation of the exposure setup including all other instruments.

1. Before starting an exposure using the AES, connect the system to several instruments to measure aerosol characteristics; these are measured in a side stream just before the aerosols enter the cabinet.

NOTE: A flow splitter is used to connect the side stream to the exposure characterization equipment. Generally, the following equipment is used: scanning mobility particle sizer (SMPS), optical particle sizer (OPS), condensation particle counter (CPC), tapered element oscillating microbalance (TEOM). The SMPS and OPS measurements are performed $1 x$ per hour and use the same tubing from the flow splitter. The CPC and TEOM perform continuous measuring and data from both are logged on a Squirrel model 2020 data logger. In addition, gravimetric mass concentration is determined using a microbalance in controlled relative humidity $(40-70 \%)$ and temperature $\left(21-23^{\circ} \mathrm{C}\right)$ conditions. Teflon filters are weighed before and after each exposure to confirm the exposure concentration. To capture the exhaust, a HEPA filter is used. The setup including engineered nanomaterial (ENM) suspensions and nebulizer are all placed in a flow cabinet to prevent any exposure to people. The AES can be used for testing many different types of ENMs, including metals and metal oxides.

2. Prepare a nanomaterial suspension shortly before exposure. Usually a $1 \%$ suspension is prepared as a stock solution. For example, suspend $100 \mathrm{mg}$ of nanomaterial in $10 \mathrm{~mL}$ of pure water.

NOTE: For DQ12 exposure, $300 \mathrm{mg}$ is used to achieve about $2 \mu \mathrm{g} / \mathrm{cm}^{2}$. This amount can be used in a single exposure or divided over repeated exposures (e.g., $300 \mathrm{mg}$ is suspended in $30 \mathrm{~mL}$ for a single exposure or $21.5 \mathrm{mg}$ is suspended in $2.15 \mathrm{~mL}$ freshly every day for 3 weeks of repeated exposure). The suspension is freshly prepared on each exposure day. The particle suspension is sonicated for 16 min using probe sonication. The volume of the $1 \%$ stock solution is adjusted to a total volume of $100 \mathrm{~mL}$ by adding pure water.

3. Put the ENM suspension in a small bottle with a cap and a magnetic stirrer to prevent settling of the particles. Connect the bottle to a peristaltic pump via a small tube and adjust the flow to $25 \mathrm{~mL} / \mathrm{h}$.

4. Connect the peristaltic pump to a spray nozzle and adjust the settings to allow continuous aerosolization.

5. The spray nozzle is mounted to a $60 \mathrm{~cm}$ long aluminium tube (mixing chamber, diameter $15 \mathrm{~cm}$, heated to $60{ }^{\circ} \mathrm{C}$ ). The setup is connected to the AES via a 1.5 meter long copper tube (diameter $15 \mathrm{~cm}$ ). On top of the AES an impactor removes all aerosols larger than $2.5 \mu \mathrm{m}$.

6. Connect the spray nozzle to 3 bar compressed air through two mass flow controllers (MFC) to allow nebulization of suspensions. One flow of $14 \mathrm{~L} / \mathrm{min}$ is used for the spray nozzle, the other MFC for mixing of the air in the tube.

7. The day before the start of the exposure, turn on the AES to allow the cabinet to reach a temperature of $37^{\circ} \mathrm{C}$.

8. Turn on the air flow and the humidity in the cabinet $2 \mathrm{~h}$ before start of the exposure, to reach $85 \%$ humidity. Turn on the heating of the exposure chambers in which the inserts with the cells will be placed.

9. Turn on the quartz microbalance (QCM) and set the initial value at 0 using the software. Start logging. Every $10 \mathrm{~s}$ the mass is measured and expressed as $\mathrm{ng} / \mathrm{cm}^{2}$.

10. Warm the cell culture media to $37^{\circ} \mathrm{C}$ in a water bath $(\sim 20-30 \mathrm{~min})$.

\section{Preparing Calu-3 cells for exposure}

NOTE: For a typical ALI exposure using the AES, 15-20 inserts with a confluent cell layer are needed. These consist of 3 clean air controls, 3 incubator controls that will be handled similarly to the other inserts without exposure in the AES, 6-8 inserts for aerosol exposure (depending on the use of 0,1 , or 2 microbalances), 1-3 inserts for control measurements (such as maximum LDH release), and 3 spare inserts in case the TEER of some of the inserts is not sufficient. The cells should have a TEER of $>1,000 \Omega \times \mathrm{cm}^{2}$ to continue.

1. On the first day of exposure, wash cells $1 \mathrm{x}$ with $\mathrm{CCM}$, check cell morphology, and measure the TEER of the cell model using a Voltohmmeter. The cells should form a tight monolayer without gaps.

2. Put $2 \mathrm{~mL} / 1 \mathrm{~mL}$ of HEPES buffered CCM without FCS to the basolateral side of 6 well/12 well plates and transfer the culture inserts with cells to the new plates.

NOTE: During exposure, no $\mathrm{CO}_{2}$ is present in the AES. Therefore, HEPES buffered culture medium (25 mM HEPES) is used during transport and exposure. This medium is used for both the exposed cells in the AES as well as the incubator control cells.

3. In case the time to transport the cell cultures to the AES is more than $5 \mathrm{~min}$, put the cells in a portable incubator of $37^{\circ} \mathrm{C}$ during transport. 


\section{Handling the AES during an exposure}

1. At the AES, fill the exposure modules with HEPES-buffered CCM without fetal calf serum (FCS). The amount of CCM depends on the unit used, and the type of cell culture insert. Keep in mind that to keep cells at the ALI, the medium should reach the bottom of the membrane, but should not leak on top of the membrane. When using 6 well inserts, add $6 \mathrm{~mL}$ of HEPES-buffered CCM to the exposure modules.

2. Transfer the inserts with cells from the plates to the exposure modules using sterile tweezers. Check that there are no air bubbles at the basolateral side of the cells and remove any CCM on the apical side of the inserts. In case there are some air bubbles at the basolateral side, gently turn the inserts using the sterile tweezers until they are removed. Keep the plates containing CCM in an incubator for transfer after an exposure.

3. Use the touchscreen display to choose exposure duration, air flow rate, and electrostatic deposition enhancement. The display can also be used to check humidity and temperature. Usually, an exposure duration of $4 \mathrm{~h}$ is chosen, with a flow rate of $50 \mathrm{~mL} / \mathrm{min}$ on the inserts at $37{ }^{\circ} \mathrm{C}$ and $85 \%$ humidity.

NOTE: The modules in the first level (Figure 1) are used for clean air exposure; inserts in this level are used as clean air exposure controls. The other modules in the second and third level can be used for aerosol exposure, including two modules for quartz crystal microbalances (QCM) to measure deposition online.

4. The leak test should be conducted before starting exposure. The leakage needs to be less than $5 \mathrm{~mL} / \mathrm{min}$. Follow the instructions on the AES display. When the leak test has finished, the exposure can be started. In case of a leak, the tubing should be checked.

5. At the end of exposure, open the door of an AES module, open the exposure modules, place the cell culture inserts back to the cell culture plates, and transfer the plates to the portable incubator.

6. Collect the media from the modules (i.e., exposed samples) and from the plates (i.e., incubator controls) for later analysis, such as lactate dehydrogenase (LDH) measurement.

7. Back at the cell culture lab, transfer the cell culture inserts to plates filled with fresh standard CCM. Put the cell culture plates in the incubator until the next exposure or until analysis.

8. After the final exposure day, put the inserts in the incubator until the next day.

9. At the day after the final exposure, add CCM to the apical side to measure TEER using a Voltohmmeter. Collect both the apical and the basolateral CCM separately for analysis of cytokines.

10. Remove all CCM and perform a cell viability assay by adding, for example, a proliferation reagent to the apical side.

\section{Cleaning the AES}

1. Fill the exposure modules with water, wait for $1 \mathrm{~min}$, and remove the water. Next, fill the modules with $70 \%$ ethanol, leave it for 10 min, and remove the ethanol. Clean the exposure trumpets also with $70 \%$ ethanol.

2. Stop the $85 \%$ humidity control, but leave the temperature of the cabinet at $37^{\circ} \mathrm{C}$ for the next experiment.

\section{Representative Results}

This article provides a method for culturing and exposing human bronchial epithelial cells at the ALI that mimics realistic, repeated inhalation exposure conditions that can be used for toxicity testing. Characteristics of both the cell model and of the exposure system are essential for achieving a realistic inhalation exposure model that can be used for repeated exposures. Results on these characteristics are shown below.

\section{Cell model requirements and selection}

When selecting a suitable cell model, the following characteristics must be taken into account:

1. The cell model should be able to form a confluent monolayer with functioning tight junctions to mimic the lung barrier.

2. The cell model should show optimal performance when exposed repeatedly to conditioned (temperature and humidity) air.

3. The cell model should respond to an exposure.

This study started with four different human bronchial epithelial cell lines: $16 \mathrm{HBE}$, Calu-3, H292, and BEAS-2B. These are all widely used for toxicity testing of nanomaterials and chemicals. Of the four cell lines, only the Calu-3 cells fulfilled all the above requirements. The cells formed a monolayer with tight junctions (Figure 3) that remained a stable barrier over time as measured by TEER, whereas the other cell lines either did not form a barrier or showed a drop in barrier function when cultured at the ALI (Figure 4). In addition, H292 and BEAS-2B tended to overgrow into multiple cell layers when cultured for a longer time period. Traditional submerged cell culturing and ALI culturing differed greatly, because at the ALI nutrients were only available from the basolateral side and the cells were exposed to dry conditions at the apical side. These conditions can cause stress to the cell models, which could be observed by measuring the cell viability over time. Cell lines $16 \mathrm{HBE}, \mathrm{H} 292$, and BEAS-2B all showed an increased LDH release when cultured at the ALI, while Calu-3 cells showed only a slight LDH release (Figure 5).

Next, the response of the Calu-3 model to substances was tested. As a positive control substance, LPS was administered via nebulization to the apical side of the model. The deposited dose was $0.25 \mu \mathrm{g} / \mathrm{cm}^{2}$. The Calu-3 cells showed a reaction to lipopolysaccharide (LPS) by an increase in $\mathrm{LDH}$ release and in tumor necrosis factor alpha (TNF- $\alpha$ ) release (Figure 6).

Finally, the Calu-3 monolayer was exposed to quartz silica (DQ12) nanomaterials (IOM, Edinburgh). Crystalline silica can induce silicosis and may also cause lung tumors. Therefore, the International Agency for Research on Cancer (IARC) has classified crystalline silica as a Group I human carcinogen 20 . The mechanism of action of crystalline silica is thought to be via the induction of persistent inflammation caused by its reactive surface ${ }^{21,22,23}$. Several in vivo studies in both rats and mice report the induction of inflammation and histopathology changes, including tumors and fibrosis, after inhalation exposure to crystalline silica ${ }^{24,25,26,27,28,29}$. These effects are all observed after repeated exposure and/or long-term follow-up. The Calu-3 model was used to investigate whether the observations from the in vivo studies could be mimicked using an in vitro model that could be exposed repeatedly at the ALI. 
Calu-3 cells were exposed for 3 consecutive weeks, 5 days per week, $4 \mathrm{~h}$ per day to DQ12. The deposited dose was measured using a QCM. The average deposited dose was $120 \mathrm{ng} / \mathrm{cm}^{2}$ per day, with a cumulative dose of $1.6 \mu \mathrm{g} / \mathrm{cm}^{2}$, similar to the doses inducing an effect in vivo. Other particle characteristics are shown in Table 1. After 3 weeks of exposure, DQ12 induced no significant effects in TEER, cell viability, and monocyte chemoattractant protein 1 (MCP-1) release, compared to the clean air controls (Figure 7). As more toxicity of DQ12 was expected based on the in vivo data, the reactivity of the particles was checked using an acellular assay according to the protocol optimized within the EU-project GRACIOUS (deliverable 5.3). The reactivity of the DQ12 batch was lower than expected (Figure 8), orders of magnitudes lower compared to the positive control particles carbon black (CB). This lack of reactivity might explain the absence of a toxicity response in the Calu-3 model.
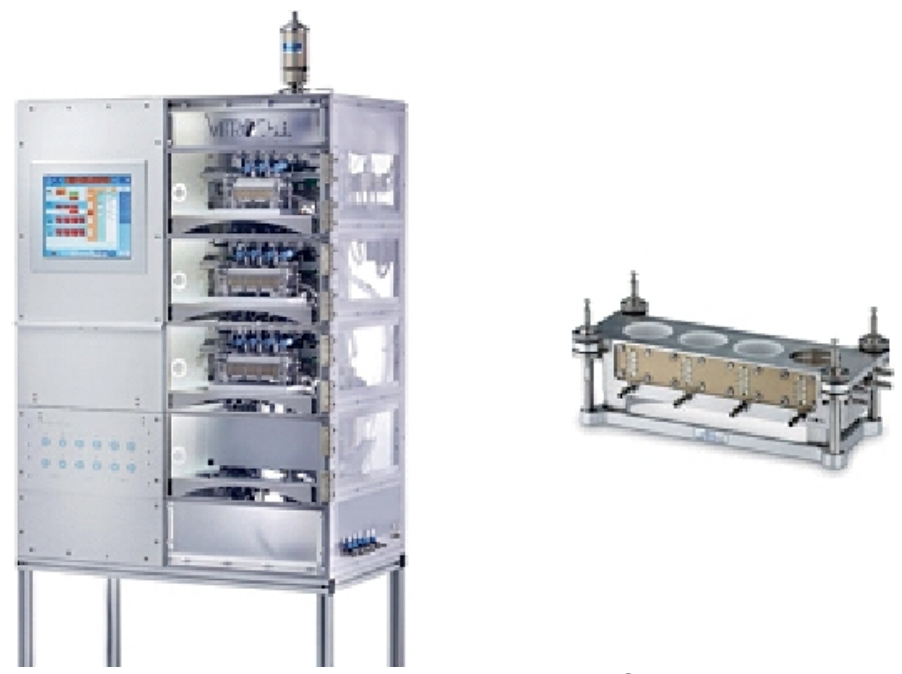

Figure 1: The automated exposure station (AES).

The left figure shows the outside of the cabinet with the touch panel. The AES has three levels with exposure modules: the top level for clean air exposures, and the middle and bottom level for aerosol exposures. The right figure shows the exposure module in which inserts with cells are placed. Please click here to view a larger version of this figure.

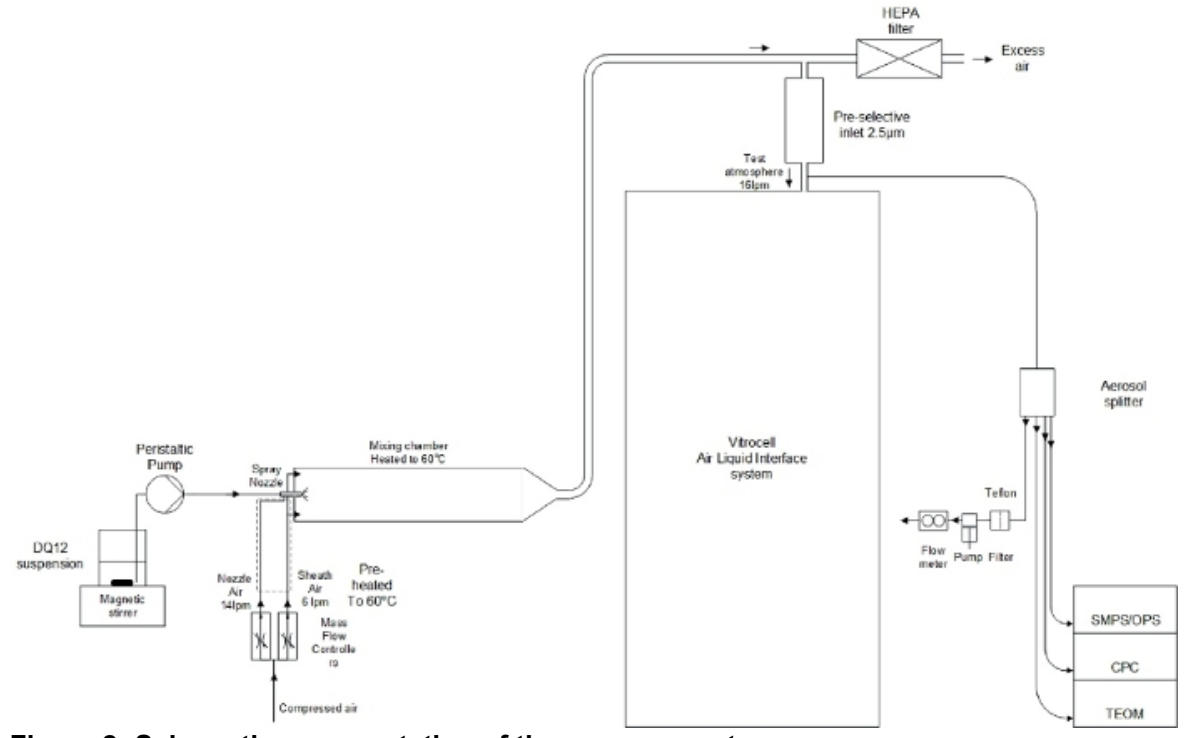

Figure 2: Schematic representation of the exposure setup.

From left to right: 1) the ENM suspension connected to the spray nozzle via a peristaltic pump; 2) Using compressed air the spray nozzle nebulizes the ENM suspension and via a mixing chamber the aerosols are led to the AES; 3 ) Just before entering the AES, aerosol characterization instruments are connected: SMPS, OPS, CPC, and TEOM. Please click here to view a larger version of this figure. 


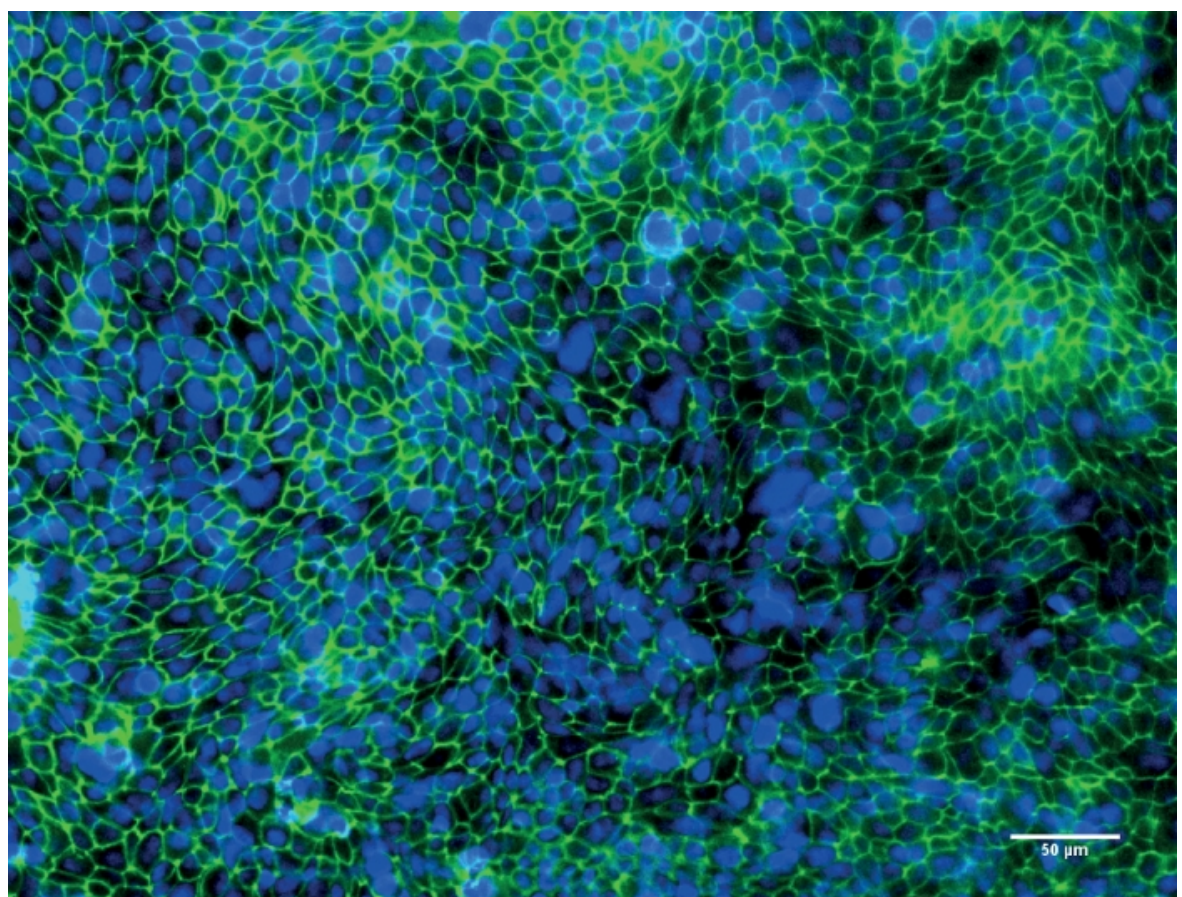

Figure 3: Representative image of Calu-3 cells after culturing at the air-liquid interface (ALI) for 10 days.

Fluorescence microscopy image of Calu-3 cells after culturing at the ALI for 10 days. Tight junction protein ZO-1 is stained in green, nuclei of the cells are stained in blue. Please click here to view a larger version of this figure.

\section{TEER values 4 different human bronchial epithelial cell lines}

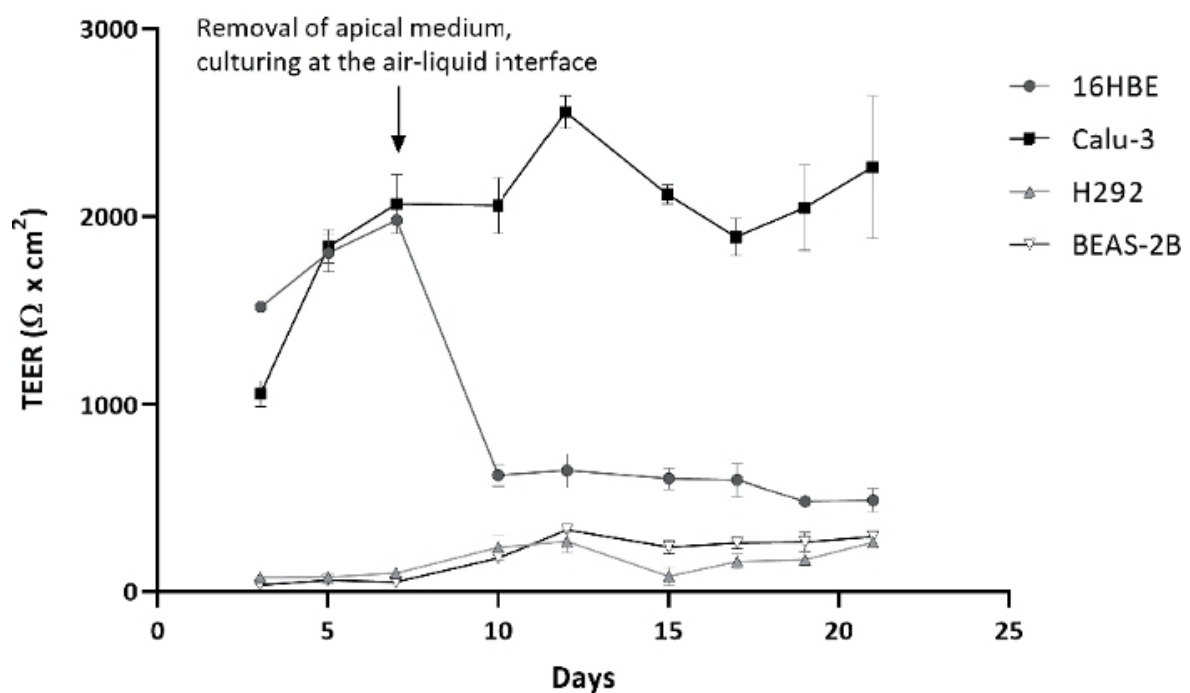

Figure 4: Transepithelial electrical resistance (TEER) of four different cell lines during a culture period of 21 days.

TEER values of $16 \mathrm{HBE}$, Calu-3, H292, and BEAS-2B when cultured for 21 days: first 7 days submerged, followed by 14 days at the ALI. TEER values were corrected for the background resistance of the insert and multiplied by the surface area of the insert. The symbols and error bars represent the average value and standard deviation of six inserts. Please click here to view a larger version of this figure. 


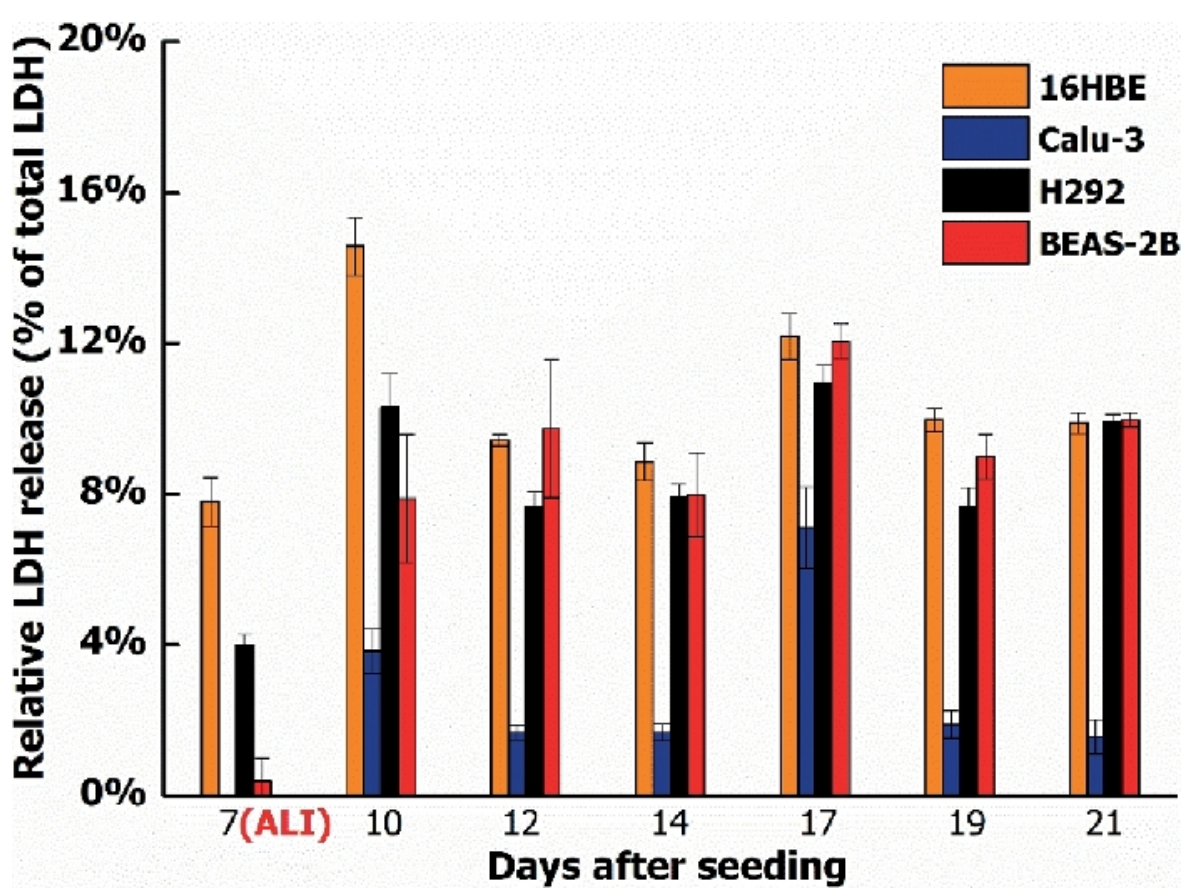

Figure 5: LDH release of four different cell lines during a culture period of 21 days.

LDH release of $16 \mathrm{HBE}$, Calu-3, H292, and BEAS-2B when cultured for 21 days: 7 days submerged, followed by 14 days at the ALI. LDH values shown are relative to the maximum LDH release per cell type. The symbols and error bars represent the average value and standard deviation of five inserts. Please click here to view a larger version of this figure.
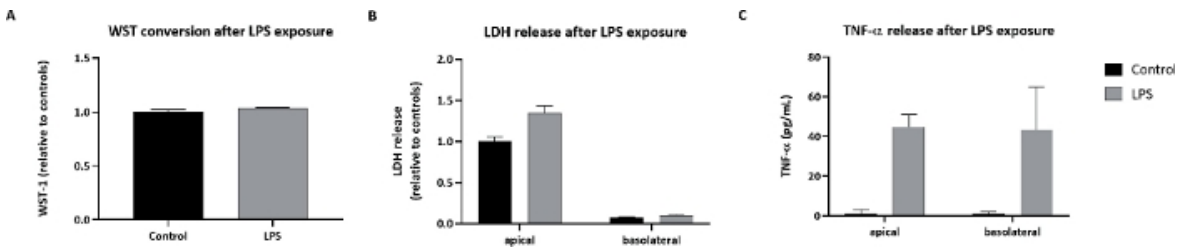

Figure 6: Cellular effects in Calu-3 cells exposed to LPS.

Calu-3 cells were exposed via cloud nebulization to $0.25 \mu \mathrm{g} / \mathrm{cm}^{2}$ LPS. (A) The WST-1 conversion. (B) LDH release. (C) TNF- $\alpha$ release after LPS exposure. The symbols and error bars represent average values and standard deviations of three inserts. Please click here to view a larger version of this figure.
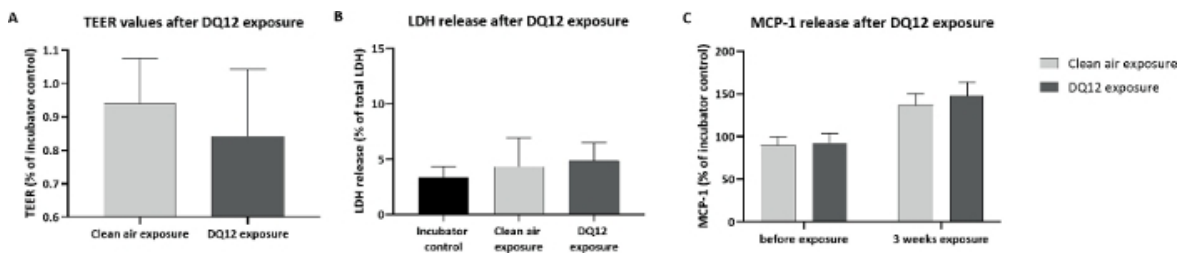

Figure 7: Cellular effects in Calu-3 cells exposed to DQ12 nanomaterials.

Calu-3 cells were exposed for 3 weeks ( $4 \mathrm{~h}$ per day, 5 days per week) to DQ12 nanomaterials, about $120 \mathrm{ng} / \mathrm{cm}^{2}$ per day, cumulative dose of $1.6 \mu \mathrm{g} / \mathrm{cm}^{2}$. (A) TEER values. (B) LDH release. (C) MCP-1 release after DQ12 exposure. All symbols and error bars represent average values and standard deviations of three inserts for the controls and six inserts for the DQ12 exposure. Please click here to view a larger version of this figure. 


\section{Reactivity of DQ12}

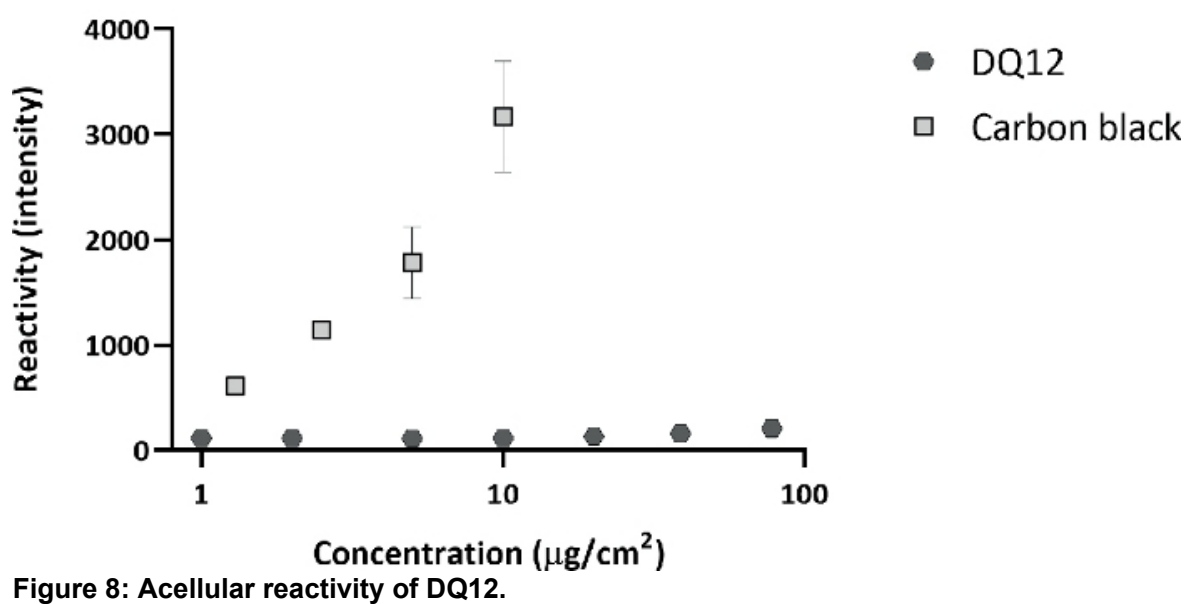

DQ12 was incubated with a 2',7'-Dichlorofluorescein Diacetate (DCFH-DA) probe to detects its surface reactivity. As a positive control, carbon black (CB) particles were included. Compared to CB, DQ12 has very low surface reactivity. Please click here to view a larger version of this figure.

\begin{tabular}{|l|l|l|l|l|}
\hline Particle mass $\left(\mathbf{\mu g} / \mathbf{m}^{3}\right)$ & Particle number $\left(\# / \mathbf{c m}^{3}\right)$ & Mobility particle size $(\mathbf{n m})$ & $\begin{array}{l}\text { Geometric standard } \\
\text { deviation }\end{array}$ & Optical particle size $(\boldsymbol{\mu m})$ \\
\hline $2969(418)$ & $83983(10215)$ & 66.6 & 2.5 & $1.1(1.3)$ \\
\hline
\end{tabular}

Table 1: DQ12 exposure characteristics. Values are shown as average with standard deviation in brackets.

\section{Discussion}

This paper describes a method for culturing human bronchial epithelial cells under ALI and exposing this bronchial model to aerosols or gases. The advantage of using Calu-3 cells is that they form tight junctions, remain a monolayer, are able to withstand the air flow, and can be cultured for weeks at the ALI, unlike many other cell types (e.g., 16HBE, H292, and BEAS-2B). Using the VITROCELL ${ }^{\circledR}$ automated exposure station (AES) has the advantage that the cells can be exposed under realistic and relevant conditions as low concentrations can be applied using a continuous airflow.

Continued flow systems, such as the AES, have advantages compared to using cloud systems $\mathrm{s}^{3,32}$, which use a single nebulization of a suspension. The continuous flow is more realistic and many variables like flow rate, humidity, and temperature are controlled. In addition, deposition can be enhanced using an electrical field. Finally, aerosol characteristics like size, number concentration, and mass are monitored online. A disadvantage is that continued flow systems are more complex compared to cloud systems. Therefore, it is important to run preparatory experiments that focus on the particle characteristics of the aerosol and the delivered dose on the insert. The initial starting concentration of the particles and the AES settings can then be adjusted to achieve the desired dose on the cells ${ }^{33}$. Depending on the type of particles being tested, the aerosol generation method can differ. The use of electrostatic deposition depends on the particle type and works best for metallic particles. For particles with a positive surface charge a negative electrostatic field should be applied and vice versa.

Selection of exposure concentrations can be difficult for any air-liquid exposure experiment. For the DQ12 exposures, the aim was to achieve a total cumulative dose of $1 \mu \mathrm{g} / \mathrm{cm}^{2}$ after 3 weeks exposure, 5 days per week, $4 \mathrm{~h}$ per day. This dose is similar to doses that induced an effect in vivo $^{21,25,27,32,33}$. When performing the exposures, there was some variation between different exposure days. Although the actual deposited dose of $1.6 \mu \mathrm{g} / \mathrm{cm}^{2}$ is higher than the $1 \mu \mathrm{g} / \mathrm{cm}^{2}$ that was aimed for, the dose might have been too low to observe effects in the Calu-3 model. Only minor differences in TEER, viability, and cytokine response were observed between the clean air exposure and the DQ12 exposure, and these differences were not statistically significant. An explanation for the observation that DQ12 exposure for 3 weeks did not induce significant effects in the Calu-3 cells is that macrophages were lacking from the Calu-3 model. Possibly, after DQ12 uptake macrophages produce proinflammatory cytokines that may affect Calu-3 cells. Another explanation is that the DQ12 batch that was used for the experiments was not as reactive as expected. When using LPS as a positive control substance, Calu-3 does show a response, as measured by an increase in LDH release and an increase in TNF- $\alpha$ release. This indicates that the model can detect toxicity.

The Calu- 3 cell model has many advantages, as discussed in the results section. Moreover, when cultured for a longer time at the ALI, the Calu-3 cells can grow cilia/cilia-like structures ${ }^{13}$ and produce mucus ${ }^{11,12,13}$. Despite these advantages, the model has limitations with respect to its physiological relevance. The Calu- 3 cell lines originates from an adenocarcinoma, whereas $16 \mathrm{HBE}$ and BEAS-2B originate from healthy tissue. Unfortunately, the latter two are not suitable for repeated ALI exposure as they do not remain a stable monolayer over time. Another limitation of the Calu-3 model is that it only represents a single cell type. In the human lung, multiple cell types that interact and respond differently to exposure are present. Inhaled particles will deposit in different regions of the lungs depending on their aerodynamic size. This is where the particles contact the epithelial cell barrier, as mimicked by the Calu-3 model. In the human lung, alveolar macrophages are attracted to the particles, engulf them, and clear them from the lungs. Macrophages also play an essential role in the inflammation response to particle exposure. Therefore, efforts are being made to extend the Calu-3 model by adding primary macrophages to mimic the lung barrier more closely. The disadvantage of the macrophages is that they remain viable only for about 7 days when cultured on top of Calu- 3 cells at the ALI. Therefore, 
macrophages should be readded weekly to transform the current Calu-3 model into a coculture model. The optimization of the coculture protocol is currently ongoing.

Given the above, the Calu-3 bronchial model is a suitable model for repeated exposure to aerosols of partly soluble substances such as chemicals from cigarette smoke and LPS. These soluble substances induce significant increases in cytokine responses in the Calu- 3 cells. For testing insoluble particles such as diesel exhaust and DQ12, a coculture model is needed, because the macrophages play a crucial role in the induction of effects by particle exposure.

For the exposures described, insert membranes with $3.0 \mu \mathrm{m}$ pores were used. The main reason for choosing this type of insert is that it is possible to test the translocation of nanomaterials. When using smaller $0.4 \mu \mathrm{m}$ pore size, particle agglomerates will not be able to cross the insert membrane. The disadvantage of using a large pore size is that the cells need a longer time to grow confluent and that it is more difficult to visualize the morphology of the cells using light microscopy. To check that the cells do form a confluent monolayer, the TEER should be $>1,000$ $\Omega \times \mathrm{cm}^{2}$ before starting an exposure.

Taken together, the Calu- 3 bronchial model presented here is suitable to use for repeated exposure to aerosols, at least up to 3 weeks. The model can withstand being cultured and exposed via a continuous airflow and is capable to detect toxicity to the bronchial epithelium.

\section{Disclosures}

The authors have nothing to disclose.

\section{Acknowledgments}

This work is funded by EU-project PATROLS (Physiologically Anchored Tools for Realistic nanomaterial hazard aSsessment) and the Dutch Ministry of Health, Welfare and Sport (project V/050012). We would like to thank Dr. Yvonne Staal and Dr. Jan van Benthem for critically reviewing the manuscript.

\section{References}

1. Geiser, M., Jeannet, N., Fierz, M., Burtscher, H. Evaluating Adverse Effects of Inhaled Nanoparticles by Realistic In Vitro Technology. Nanomaterials (Basel). 7 (2), E49 (2017).

2. Herzog, F. et al. Mimicking exposures to acute and lifetime concentrations of inhaled silver nanoparticles by two different in vitro approaches. The Beilstein Journal of Nanotechnology. 5, 1357-1370 (2014).

3. Lenz, A. G. et al. A dose-controlled system for air-liquid interface cell exposure and application to zinc oxide nanoparticles. Particle and Fibre Toxicology. 6, 32 (2009).

4. Paur, H.R. et al. In-vitro cell exposure studies for the assessment of nanoparticle toxicity in the lung-A dialog between aerosol science and biology. Journal of Aerosol Science. 42 (10), 668-692 (2011).

5. Lacroix, G. et al. Air-Liquid Interface In Vitro Models for Respiratory Toxicology Research: Consensus Workshop and Recommendations. Applied in vitro Toxicology. 4 (2), 91-106 (2018).

6. Loret, T. et al. Air-liquid interface exposure to aerosols of poorly soluble nanomaterials induces different biological activation levels compared to exposure to suspensions. Particle and Fibre Toxicology. 13 (1), 58 (2016).

7. Zhu, Y., Chidekel, A., Shaffer, T. H. Cultured human airway epithelial cells (calu-3): a model of human respiratory function, structure, and inflammatory responses. Critical Care Research and Practice. 2010 (2010).

8. Braakhuis, H. M. et al. Progress and future of in vitro models to study translocation of nanoparticles. Archives in Toxicology. 89 (9), 1469-1495 (2015).

9. Ren, H., Birch, N. P., Suresh, V. An Optimised Human Cell Culture Model for Alveolar Epithelial Transport. PLoS One. 11 (10), e0165225e0165225 (2016).

10. Srinivasan, B. et al. TEER measurement techniques for in vitro barrier model systems. Journal of Laboratory Automation. 20 (2), $107-126$ (2015).

11. Papazian, D., Wurtzen, P. A., Hansen, S. W. Polarized Airway Epithelial Models for Immunological Co-Culture Studies. International Archives of Allergy and Immunology. 170 (1), 1-21 (2016).

12. Jeong, M. H. et al. In vitro model for predicting acute inhalation toxicity by using a Calu-3 epithelium cytotoxicity assay. Journal of Pharmacological and Toxicological Methods. 98, 106576 (2019).

13. Grainger, C. I., Greenwell, L. L., Lockley, D. J., Martin, G. P., Forbes, B. Culture of Calu-3 cells at the air interface provides a representative model of the airway epithelial barrier. Pharmacology Research. 23 (7), 1482-1490 (2006).

14. Harcourt, J. L., Caidi, H., Anderson, L. J., Haynes, L. M. Evaluation of the Calu-3 cell line as a model of in vitro respiratory syncytial virus infection. Journal of Virological Methods. 174 (1-2), 144-149 (2011).

15. Vitrocell. VITROCELL® Automated Exposure Station. <https://www.vitrocell.com/inhalation-toxicology/exposure-systems/automatedexposure-station $1>(2019)$

16. Mülhopt, S. et al. Toxicity testing of combustion aerosols at the air-liquid interface with a self-contained and easy-to-use exposure system. Journal of Aerosol Science. 96, 38-55 (2016).

17. Vitrocell. VITROCELL $®$ Cloud for the exposure to liquid aerosols. <https://www.vitrocell.com/inhalation-toxicology/exposure-systems/vitrocellcloud-system> (2019).

18. Endes, C. et al. An in vitro testing strategy towards mimicking the inhalation of high aspect ratio nanoparticles. Particle and Fibre Toxicology. 11 (1), 40 (2014)

19. Lenz, A. G. et al. Efficient bioactive delivery of aerosolized drugs to human pulmonary epithelial cells cultured in air-liquid interface conditions Am J Respir Cell Mol Biol. 51 (4), 526-535 (2014). 
20. Borm, P. J., Tran, L., Donaldson, K. The carcinogenic action of crystalline silica: a review of the evidence supporting secondary inflammationdriven genotoxicity as a principal mechanism. Critical Reviews in Toxicology. 41 (9), 756-770 (2011).

21. Borm, P. J. A., Fowler, P., Kirkland, D. An updated review of the genotoxicity of respirable crystalline silica. Particle and Fibre Toxicology. 15 (1), 23 (2018).

22. Kawasaki, H. A mechanistic review of silica-induced inhalation toxicity. Inhalation Toxicology. 27 (8), 363-377 (2015).

23. Sayes, C. M. et al. Changing the dose metric for inhalation toxicity studies: short-term study in rats with engineered aerosolized amorphous silica nanoparticles. Inhalation Toxicology. 22 (4), 348-354 (2010).

24. Driscoll, K. E. et al. Pulmonary response to inhaled silica or titanium dioxide. Toxicology and Applied Pharmacology. 111 (2), 201-210 (1991).

25. Muhle, H., Kittel, B., Ernst, H., Mohr, U., Mermelstein, R. Neoplastic lung lesions in rat after chronic exposure to crystalline silica. Scandinavian Journal of Work, Environment and Health. 21 (Suppl 2) 27-29 (1995).

26. Peeters, P. M. et al. Silica-induced NLRP3 inflammasome activation in vitro and in rat lungs. Particle and Fibre Toxicology. 11, 58 (2014).

27. Reuzel, P. G., Bruijntjes, J. P., Feron, V. J., Woutersen, R. A. Subchronic inhalation toxicity of amorphous silicas and quartz dust in rats. Food and Chemical Toxicology. 29 (5), 341-354 (1991).

28. Arts, J. H., Muijser, H., Duistermaat, E., Junker, K., Kuper, C. F. Five-day inhalation toxicity study of three types of synthetic amorphous silicas in Wistar rats and post-exposure evaluations for up to 3 months. Food and Chemical Toxicology. 45 (10), 1856-1867 (2007).

29. Cho, W. S. et al. Inflammatory mediators induced by intratracheal instillation of ultrafine amorphous silica particles. Toxicology Letters. 175 (1-3), 24-33 (2007).

30. Herzog, F. et al. Exposure of silver-nanoparticles and silver-ions to lung cells in vitro at the air-liquid interface. Particle and Fibre Toxicology. 10 (1), $11(2013)$.

31. Mülhopt, S., Diabate, S., Krebs, T., Weiss, C., Paur, H. R. Lung toxicity determination by in vitro exposure at the air liquid interface with an integrated online dose measurement. Journal of Physics: Conference Series. 170, 012008 (2009).

32. van Ravenzwaay, B. et al. Comparing fate and effects of three particles of different surface properties: nano- $\mathrm{TiO}(2)$, pigmentary $\mathrm{TiO}(2)$ and quartz. Toxicology Letters. 186 (3), 152-159 (2009).

33. Henderson, R. F. et al. A comparison of the inflammatory response of the lung to inhaled versus instilled particles in F344 rats. Fundamental and Applied Toxicology. 24 (2), 183-197 (1995). 